\title{
Contents, Vol. 37, 1917
}

Inhaltsverzeichnis.

Original-Arbeiten. seit $\beta$

Bach, H., Erwiderung auE Dr. Schanz' Arbeit: ,,Lichtbehand-

lung bei Augenleiden" . . 244

Birch-Hirschfeld, G., Eine einfache Methode zur Bestimmung

der Sehschärfe bei Simulation und Übertreibung . . . 289

Bourquin, J., Die angeborene Melanose des Auges. (Hierzu

Taf. VII-VIII) . 129, 294

Bruckner, A., Zur Kenntnis des sogenannten willkür lichen

Nystagmus 184

Cords, B., Fremdkörperextraktion aus dem Augapfel unter

Leitung des Röntgenschirmes. (Hierzu Taf el V-VI) . 67

Deidschmann, B., Das traumatische Glaukom 318

Frieberg, T., Über die Mechanik der Tränenableitung mit beson-derer Hinsicht auf die

Ergebnisse der neueren Tränen-sackoperationen 42, 211, 324

Gilbert, W., Über Rheumatismus, rheumatische und metasta-

tische Regenbogenhautentzündung 174

Goldmann, B., Die'kalorische Reizung des Ohres als Mittelzur Entlarvung von Simulation einoder beiderseitigerBlindheit 77

Hesse, B., Über die Verwendung der Tierkohle bei der Be-

handlung der Ophthalmogonoblennorrhoe ....... 311

Krüclcmann E., Über die marginale Glia und die perivaskulärenLymphbahnen der

Netzhaiitkapillaren. (Hierzu Taf elI-III) 1

v. Liebermann, L., jr., Zur Technik der orbitalen Lokal-

anästhesie bei der Enukleation 194

Lüdemann, A., Hydrodiaskop oder Kontaktglas zur Korrektur

des Keratokonus 267

Meyer, H., Ein Apparat zur Bestimmung der Dunkeladaptation

für weiße und für farbige Lichter 198

Ohm, J., Die Abderhaldensche Blutuntersuchung bei Keratokonus, Glaukom, Retinitis

pigmentosa und Augenzitternder Bergleute 82

$$
\text { IV - }
$$

Seite

Schanz, F., Bemerkung zu der Erwiderung von Herrn San.-

Rat Dr. Hugo Bach, Bad Elster 246

Stargardt, K., Über e $1 / \frac{1}{8}$ e seltene Mißbildung am Auge. (Hierzu

Tafel IV) 25

Arthur v. Hippel † 115

Bericht über die deutsehe ophthalmologische Literatur. 
Motilitätsstörungen (1916). Von Priv.-Doz. Dr. C. H. Sattler

in Königsberg 401

Optik. Von Dr. Hugo Wolff in Berlin

247,366

Spezielle Pathologie nnd Therapie. I. Semester 1916. Von

Dr. Konrad Schrader in Gera 89

Sympathische Ophthalmie (1916. I. u. II. Semester). Von

Priv.-Doz. Dr. C. H. ßattler in Königsberg 410

Untersuchungsmethoden (1915 und 1916). Von Prof. Dr.

F. Dimmer in Wien . 391

Bericht über die ausländische ophthalmologische Literatur.

Bericht über die holländische ophthalmologische Literatur desJahres 1916. II. Hälfte. Von Prof.

Dr. W. Koster-Gzn inLeiden und Dr. G. Schouíe in Amsterdam

109

Therapeutische Umschau

119

Buchanzeigen 122

Literaturverzeichnis 124, 263

D. Druckfehlerberichtigung 128

266

Sach- und Namenregister 